\title{
Prevención y tratamiento de lesiones lumbares con herramientas físico-médicas. Una revisión sistemática.
}

\section{Prevention and treatment of lumbar injuries with physical-medical tools. A systematic review.}

\author{
Gabriel González Valero; ${ }^{a}$ José Luis Ubago Jiménez ${ }^{\mathrm{a}}$; Manuel Castro Sánchez ${ }^{\mathrm{a}^{*}}$; Inmaculada \\ García Martínez ; María Sánchez Zafra ${ }^{\mathrm{a}}$

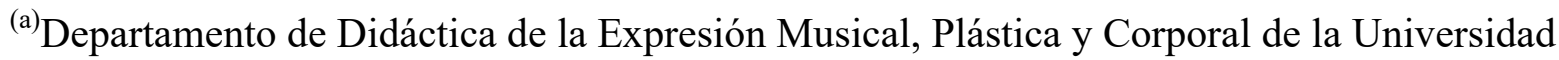 \\ de Granada (España) \\ (b) Departamento de Didáctica y Organización Escolar de la Universidad de Granada (España) \\ E-mail: manue87@,correo.ugr.es
}

\section{Resumen}

Los dolores a nivel lumbar son uno de los problemas que más afecta a la población de forma esporádica o permanente a lo largo de la vida, cuyas causas principales están relacionadas con posturas corporales incorrectas, ejercicios de impacto y rotación superior, lo cual ocasiona un deterioro del disco intervertebral. Este estudio de revisión sistemática tiene como objetivo conocer las diversas prevenciones y tratamientos para mejorar las hernias lumbosacras. Se ha utilizado como principal base de datos la Web of Science (WOS), en la que se han empleado como descriptores claves "Herniated Disc", "Disc herniation" y "Treatment" para el área de investigación "Sport Sciences". Tras la aplicación de los criterios de inclusión y codificación, el cuerpo base de esta investigación se redujo a 15 estudios. Los datos revelan que la preocupación por la temática ha ido evolucionando en sus tratamientos, pasando de la cirugía a utilizar ejercicios terapéuticos, dado los beneficios y ventajas que reportan.

\section{Palabras clave}

Hernia discal; Lumbalgia; Prevención; Tratamiento; Revisión sistemática

Para citar este artículo utilice la siguiente referencia: González-Valero, G.; Ubajo-Jiménez, J.L.; Castro-Sánchez, M.; García-Martínez, I.; Sánchez-Zafra, Mª .. (2019). Prevención y tratamiento de lesiones lumbares con herramientas físico-médicas. Una revisión sistemática. Sportis Sci J, 5 (2), $232-249$.

DOI: https://doi.org/10.17979/sportis.2019.5.2.3409

http://revistas.udc.es/ 


\section{Abstract} mayo 2019. A Coruña. España ISSN 2386-8333

Lumbar pain is one of the problems that most affects the population sporadically or permanently throughout life, whose main causes are related to incorrect body postures, impact exercises and higher rotation, which causes deterioration of the intervertebral disc. This systematic review study aims to know the various preventions and treatments to improve lumbosacral hernias. The Web of Science (WOS) has been used as the main database, in which "Herniated Disc", "Disc herniation" and "Treatment" for the research area "Sport Sciences" have been used as key descriptors. After applying the inclusion and coding criteria, the base body of this research was reduced to 15 studies. The data reveal that the concern for the subject has been evolving in their treatments, going from surgery to using therapeutic exercises, given the benefits and advantages they bring.

\section{Keywords}

Herniated disc; Low back pain; Prevention; Treatment; Systematic review

\section{Introducción}

La enfermedad intervertebral a nivel lumbar y sacro, es uno de los principales dolores que afecta a la población actualmente, ya que más del $70 \%$ de adultos han padecido estos daños en alguna ocasión de su vida, relacionados por lo general con cambios de la biomecánica de la columna vertebral, representando el $90 \%$ de intervenciones de carácter quirúrgico (Kuai, el al., 2017).

La lumbalgia es entendida como aquella que provoca dolor en la zona baja lumbar y al comienzo del sacro, pudiendo afectar a los tejidos blandos, a las estructuras de los músculos, de los tendones y a las articulaciones de la columna vertebral, y su evolución puede ser continua, o durante algunos episodios, por lo que se puede presentar de forma temporal, permanente o recidivante (Garvey, Read y Turner, 2010). Haciéndose necesario distinguir las fases de desarrollo de esta lesión (Hernández y Zamora-Salas, 2017; Su, Kusin, Li, Ahn y Ahn, 2018): (1) degeneración nuclear, siendo el núcleo pulposo el primero debido a la perdida de elasticidad, a su vez la rotura de colágeno provoca la formación de un túnel por donde fluirá el núcleo; (2) desplazamiento, por medio de la flexión se produce una disminución del volumen entre los espacios vertebrales, produciendo una tensión que empuja hacia la parte posterior el núcleo bulboso, originando una hernia discal; (3) protrusión, en esta fase el material discal es desplazado a la cavidad espinal, pudiendo provocar hernias discales a doble nivel, bilateral o extrusión masiva; (4) fibrosis,

Para citar este artículo utilice la siguiente referencia: González-Valero, G.; Ubajo-Jiménez, J.L.; Castro-Sánchez, M.; García-Martínez, I.; Sánchez-Zafra, Mª . (2019). Prevención y tratamiento de lesiones lumbares con herramientas físico-médicas. Una revisión sistemática. Sportis Sci J, 5 (2), $232-249$.

DOI: https://doi.org/10.17979/sportis.2019.5.2.3409

http://revistas.udc.es/ 
Artículo Original. Prevención y tratamiento de lesiones lumbares con herramientas físico-médicas. Una revisión sistemática. Vol. V, nº 2; p. 232-249, mayo 2019. A Coruña. España ISSN 2386-8333

iniciándose cuando aparecen procesos reparadores, donde el tejido de granulación se infiltra en el espacio discal y lo reemplaza o los últimos fragmentos nucleares pueden propasarse.

Las causas de estas lesiones son muy diversas (Al-Najjim, Shah, Rahuma y Gabbar, 2018; González-Valero, Zurita-Ortega, Puertas-Molero, Espejo-Garcés, Chacón-Cuberos y CastroSánchez, 2017), aunque vienen determinadas por la degeneración del disco vertebral, el cual permite responder correctamente a las necesidades del movimiento, cumpliendo funciones tales como la movilidad y estabilidad vertebral, en las cuales también participan los ligamentos y masas musculares paravertebrales (Herndon, Horodyski y Vincent, 2017), así como actúa como amortiguador de las cargas y choques, colaborando con el núcleo pulposo, el cual, gracias a su viscosidad reduce la magnitud de impacto Beaudette, Larson, Larson y Brown, 2016).

Dentro del ámbito deportivo, ocurre de la misma manera, provocando dolores lumbares por la realización de movimientos de flexión y extensión, así como laterización y rotación del tronco, como consecuencia de la compresión de las estructuras raquídeas dañando el nivel L5-S1 mayoritariamente, en el cual recae la mayor parte del peso de los deportistas (Winslow, Getzin, Greenberger y Silbert, 2017). La actividad que desarrollan estos sujetos diariamente, provocan situaciones que obligan a los discos vertebrales a trabajar al límite o incluso más allá de su resistencia mecánica, los cuales tendrán una mayor o menor repercusión con respecto al tipo de actividad deportiva, la intensidad con la que se realiza y la frecuencia con la que se ejecuta repetitivamente el gesto deportivo (Minhas, Kester y Hsu, 2016). En este caso los movimientos que implican una rotación forzada son los factores principales de este problema, ya que los discos no permiten generalmente una rotación superior a 2 o 3 grados. Estos dolores afectan al estado anímico de los atletas, los cuales no saben si posteriormente podrán regresar a la práctica deportiva (Pantoja, 2012; Zurita-Ortega, Olmo-Extremera, Cachón-Zagalaz, Castro-Sánchez, Ruano-Hermoso y Zurita, 2015).

Tras la gran población que se ve afectado por este problema, se ha producido un gran interés en cómo prevenir o reducir el dolor lumbo-sacral, aunque se ha de destacar que el método más utilizado ha sido el quirúrgico, actualmente se han obtenido avances sobre como la práctica de ejercicio físico se está convirtiendo en el tratamiento más idóneo, debido que mediante este se provoca una vasodilatación a nivel de los músculos durante el movimiento, así como la expulsión y destrucción de toxinas que provocan el dolor y fatiga muscular (Kovacs y Aranda, 2016). Por ello,

Para citar este artículo utilice la siguiente referencia: González-Valero, G.; Ubajo-Jiménez, J.L.; Castro-Sánchez, M.; García-Martínez, I.; Sánchez-Zafra, Mª. (2019). Prevención y tratamiento de lesiones lumbares con herramientas físico-médicas. Una revisión sistemática. Sportis Sci J, 5 (2), $232-249$.

DOI: https://doi.org/10.17979/sportis.2019.5.2.3409

http://revistas.udc.es/ 
Artículo Original. Prevención y tratamiento de lesiones lumbares con herramientas físico-médicas. Una revisión sistemática. Vol. V, nº $2 ;$ p. 232-249, mayo 2019. A Coruña. España ISSN 2386-8333

el tratamiento con ejercicio de la hernia discal o para su prevención ha de comenzarse una vez que haya pasado el dolor, así mismo, se ha de destacar que el reposo en cama no debe de exceder los tres días.

Los ejercicios planteados para dicha temática constan de tres fases, las cuales deben de estar supervisadas por un especialista de la materia, el cual corrobore la correcta ejecución, así como la evolución del programa (Bozkurt, Aktekin, Ugurlu, Balci, Sezer, y Akkus, 2017):

En la primera fase, se localizan ejercicios aeróbicos, siendo el más aconsejable caminar, debido al gran control del movimiento que se posee y por la minimización de la presión discal, seguidos posteriormente de ejercicios de estiramiento de piernas y con mucha delicadeza de la zona lumbar, evitando la flexión de tronco, ya que es la que repercute más tensión al disco vertebral (Lopes, Correia, Félix, Lopes, Cruz y Ribeiro, 2017).

La segunda fase, consiste en producir un incremento del ejercicio aeróbico, corrigiendo posturas incorrectas, así como realizar ejercicios de fuerza isométrica (Pardo, Girbés, Roussel, Izquierdo, Penick y Martín, 2018).

Y por último la tercera etapa, la cual ha de comenzarse a partir de 8-12 semanas, si se sufre esta patología, la cual debe de continuarse a lo largo de la vida. Esta etapa consiste en realizar ejercicios de estabilización lumbar, fuerza, de aumento de flexibilidad, combinándolos con ejercicios de tipo aeróbico. Ya que para prevenir o disminuir la hernia lumbar y del sacro es importante fortalecer la zona abdominal y el tono muscular en general (Atabey et al., 2014).

\section{Método de investigación}

El propósito de este estudio es el de realizar una revisión sistemática de literatura científica de alto impacto, con el objetivo de conocer los principales métodos de prevención y las pautas de actuación de las hernias en la región lumbosacra (L5-S1) en población físicamente activa. A efecto de conseguir una buena integridad y organización del manuscrito, se aboga a la declaración PRISMA para revisiones sistemáticas (Hutton et al., 2015).

\section{Procedimiento y estrategia de búsqueda}

Para la elaboración de la revisión sistemática de investigaciones científicas que abordan la temática de estudio, se ha utilizado como principal motor de búsqueda la Web of Science (WOS), apoyándonos de otros repositorios como PubMed y SCOPUS. La revisión se efectuó durante el mes

Para citar este artículo utilice la siguiente referencia: González-Valero, G.; Ubajo-Jiménez, J.L.; Castro-Sánchez, M.; García-Martínez, I.; Sánchez-Zafra, Mª . (2019). Prevención y tratamiento de lesiones lumbares con herramientas físico-médicas. Una revisión sistemática. Sportis Sci J, 5 (2), $232-249$.

DOI: https://doi.org/10.17979/sportis.2019.5.2.3409

http://revistas.udc.es/ 
Artículo Original. Prevención y tratamiento de lesiones lumbares con herramientas físico-médicas. Una revisión sistemática. Vol. V, nº 2; p. 232-249, mayo 2019. A Coruña. España ISSN 2386-8333

de noviembre de 2017, donde se han analizado y contrastado aquellos estudios que tratan la prevención y tratamiento de las hernias discales lumbosacras en población que realiza actividad físico-deportivas. Se delimitó el rango temporal de búsqueda a los últimos cinco años de publicación (2012 al 2017), incluyendo como palabras clave "Herniated Disc", "Disc herniation" "Treatment" aplicadas con los operadores de truncamiento "and" y "or". Se consideraron todos aquellos artículos publicadas en lengua anglosajona y española, obteniendo un total de 1378 estudios de carácter científico.

Así mismo, se refinó el área y dominio de investigación, considerando aquellos artículos publicados en las revistas incluidas en la categoría "Sport Sciences", reduciéndose la búsqueda a un total de 32 estudios de investigación.

Tras fijar esta población de estudio, se pasó a seleccionar la muestra de esta revisión sistemática en función de los siguientes criterios de inclusión: (1) Investigaciones científicas que presenten como variable de tratamiento o prevención las hernias discales lumbosacras (L5-S1) en población físico-deportiva; (2) Estudios que recurran a un diseño metodológico basado en la intervención y experimentación (pre-experimental, cuasi-experimental y estudio de casos); (3) Artículos que empleasen un tratamiento basado en terapias o actividades físicas para el proceso de rehabilitación o prevención de las hernias; (4) Literatura científica que muestren resultados estadísticos u observacionales que permitan analizar el problema de estudio.

Se realizó una primera lectura del título y el resumen de la población, con la intención de aplicar el primer criterio de inclusión. A posteriori, se efectuó una lectura comprensiva y sistemática del texto completo a fin de aplicar el resto de criterios inclusivos establecidos, que nos permitió desechar un total de 17 estudios que no cumplían los requisitos de inclusión conceptuales y metodológicos establecidos, ni permitían el desarrollo de la codificación fijada en la revisión sistemática.

\section{Población y muestra bibliográfica}

Partiendo de lo mostrado en el procedimiento y la estrategia de búsqueda, la población de bibliografía científica fijada para esta revisión sistemática es de 32 artículos, los cuales han sido extraídos de la Web of Science (WOS) de la Fundación Española para la Ciencia y la Tecnología. Tal y como se plasma en la figura 1, la muestra que compone el cuerpo base de estudio se 
Artículo Original. Prevención y tratamiento de lesiones lumbares con herramientas físico-médicas. Una revisión sistemática. Vol. V, nº 2; p. 232-249, mayo 2019. A Coruña. España ISSN 2386-8333

corresponde con 15 publicaciones de carácter científico, que se han seleccionados tras considerar y aplicar los criterios de inclusión y codificación.

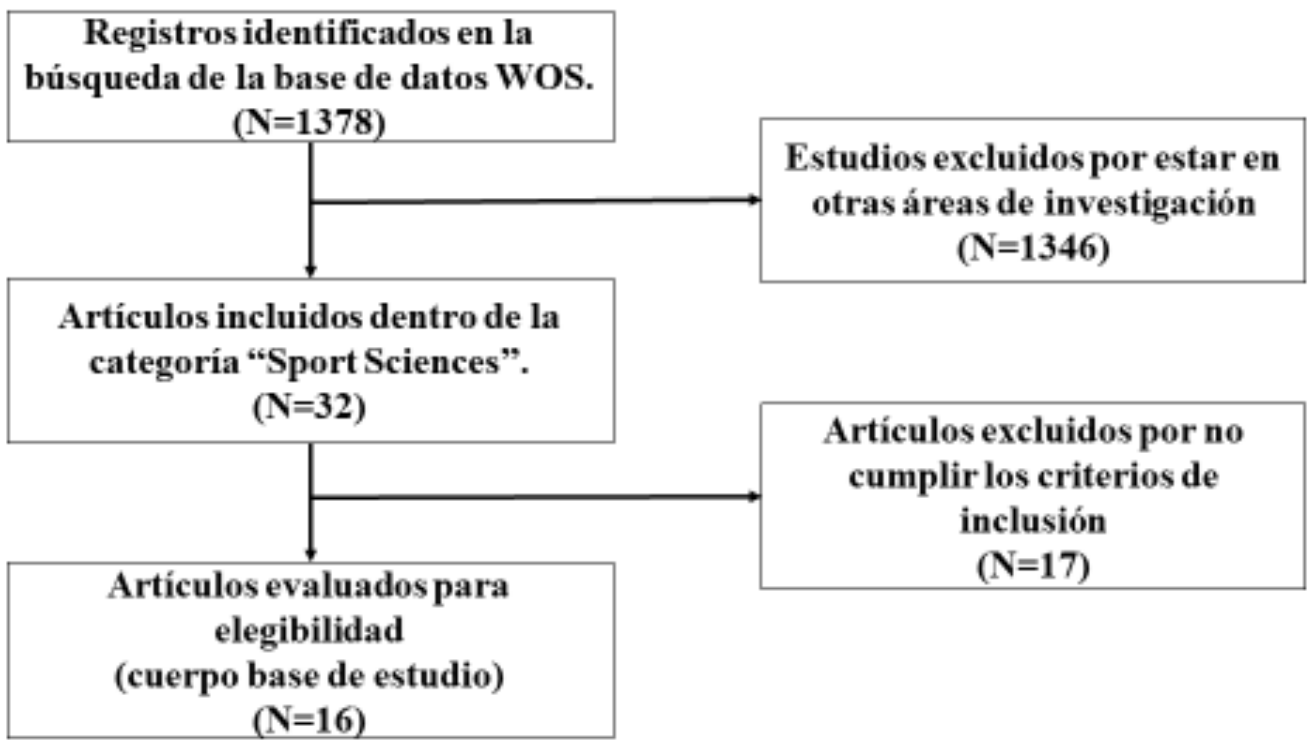

Figura 1. Diagrama de flujo de la selección de artículos del cuerpo base de investigación.

\section{Resultados}

\section{Análisis conceptual de la producción científica}

Antes de dar paso a los resultados específicos de esta revisión sistemática, cabe destacar un breve recorrido por el análisis conceptual de la producción científica según los criterios de búsqueda (Tabla 1). Se puede observar esta creación de literatura ha ido en aumento a lo largo de los últimos 5 años hasta alcanzar un total de $\mathrm{N}=1378$ estudios, donde el $99,54 \%$ de ellos han sido publicados en inglés. 
Artículo Original. Prevención y tratamiento de lesiones lumbares con herramientas físico-médicas. Una revisión sistemática. Vol. V, $n^{\circ}$. 2; p. 232-249, mayo 2019. A Coruña. España ISSN 2386-8333

Tabla 1. Evolución conceptual de la producción científica según criterios de búsqueda.

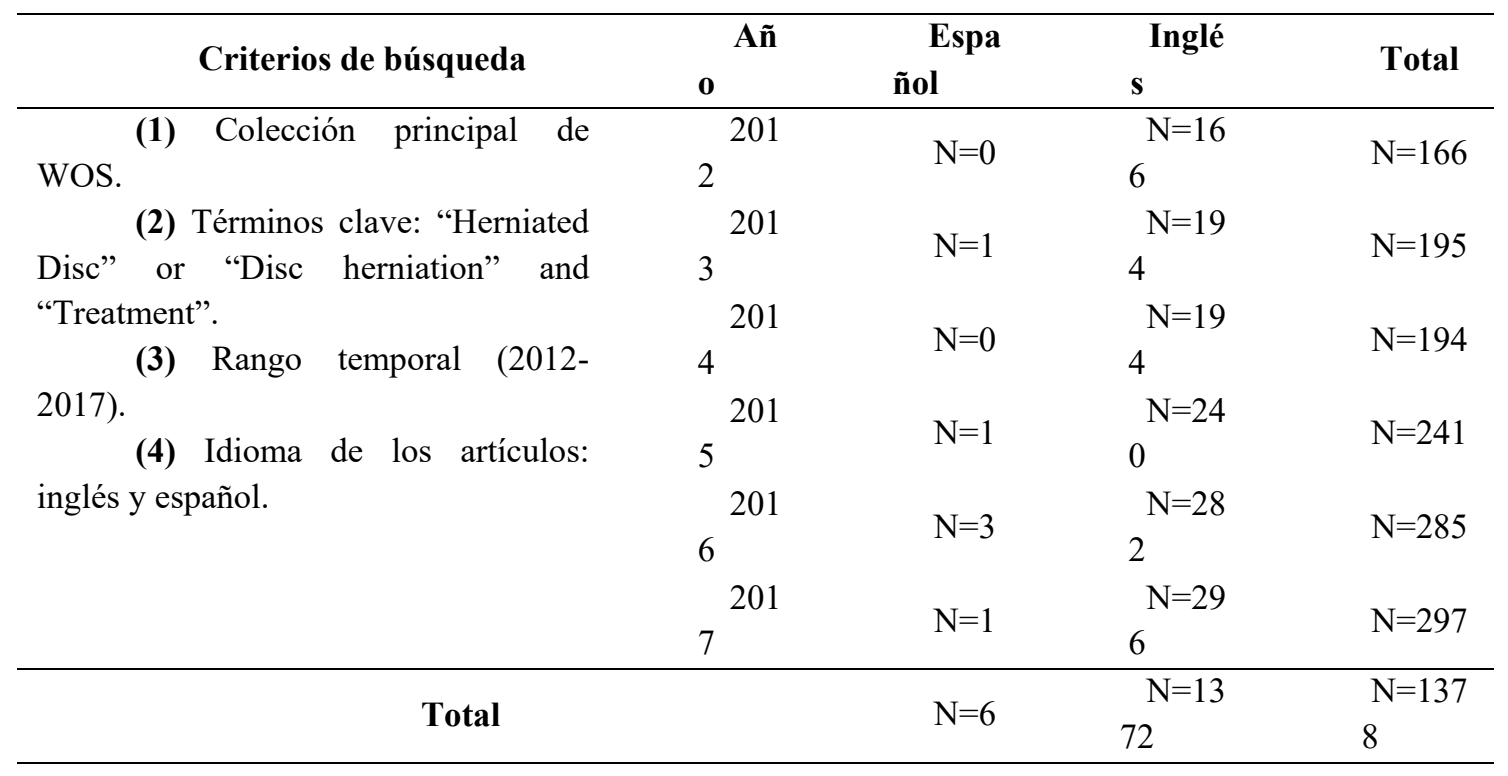

\section{Evolución de la producción científica de estudio}

En consonancia con el procedimiento de búsqueda, durante el periodo 2012-2017, para el ámbito de investigación "Sport Sciences" se registraron un total de 32 artículos de investigación científica para el tratamiento y prevención de la variable de estudio, de los cuales se han seleccionado 15 para su análisis (figura 2). Se debe destacar que el 46,87\% representa la recopilación de estudios que conforman el cuerpo base de investigación, donde al menos hay un estudio por cada año de búsqueda, además de destacar que los años 2013, 2014, 2015 y 2017 ofrecen la representatividad más alta $(\mathrm{N}=3)$. 
Artículo Original. Prevención y tratamiento de lesiones lumbares con herramientas físico-médicas. Una revisión sistemática. Vol. V, nº 2; p. 232-249, mayo 2019. A Coruña. España ISSN 2386-8333

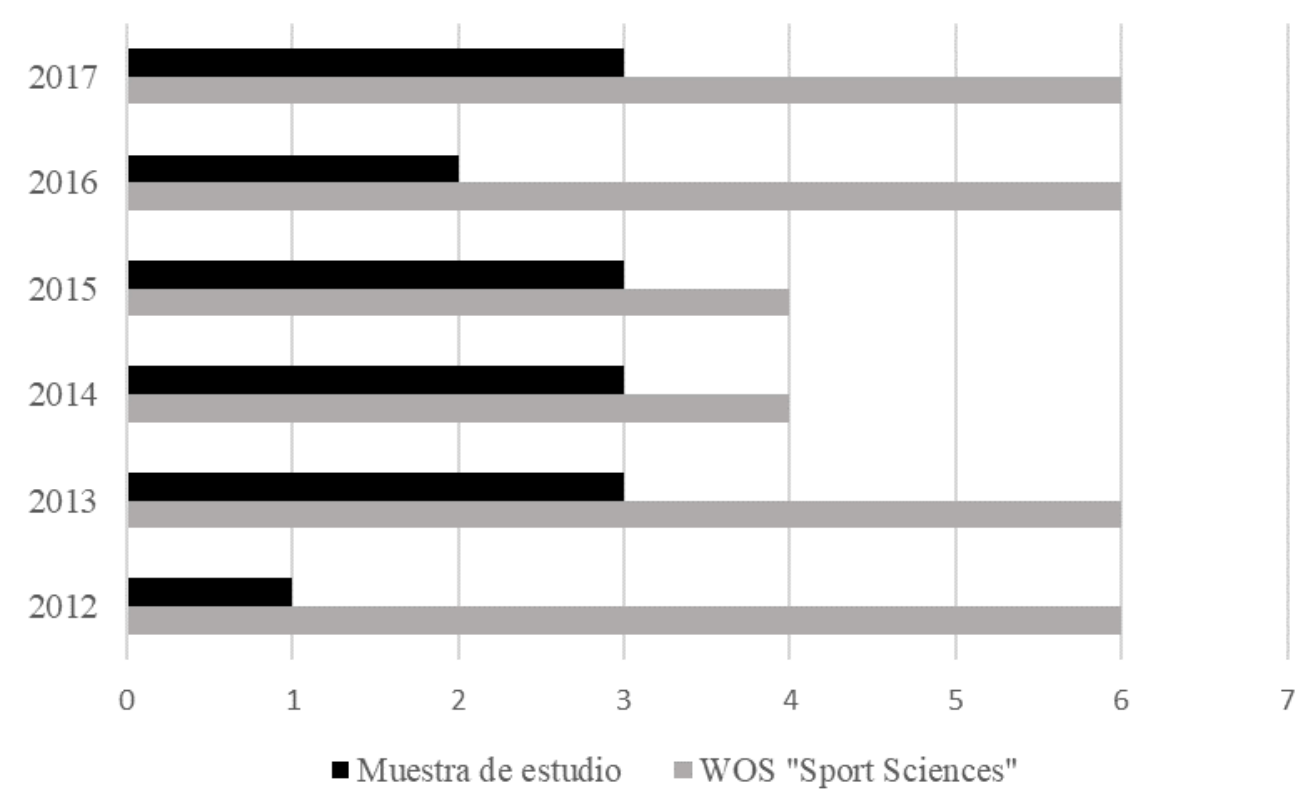

Figura 2. Evolución de la muestra de estudio según producción de la WOS.

\section{Resultados generados por la muestra de estudio}

En esta misma línea, es importante resaltar los resultados generados por los estudios que componen el cuerpo base de investigación (tabla 2), para los que se han seguido un registro basado en el siguiente proceso de codificación: (1) autores y año de publicación; (2) localización de los estudios desarrollados; (3) tipo de diseño metodológico de estudio; (4) población y muestra sobre la que se han evaluado los estudios; (5) niveles de la columna vertebral afectados; (6) tipo de tratamiento efectuado para cada estudio; (7) conclusiones arrojadas en cada investigación 
Artículo Original. Prevención y tratamiento de lesiones lumbares con herramientas físico-médicas. Una revisión sistemática. Vol. V, nº. 2; p. 232-249, mayo 2019. A Coruña. España ISSN 2386-8333 Tabla 2. Relación de estudios que tratan las hernias lumbosacras.

\begin{tabular}{|c|c|c|c|c|c|c|}
\hline Autor/es(Año) & País & Diseño del estudio & Población (Muestra) & Niveles & Tratamiento* & Conclusiones \\
\hline $\begin{array}{l}\text { Beattie, Butts, Donley y Liuzzo } \\
\text { (2014). }\end{array}$ & USA & $\begin{array}{l}\text { Pre-experimental } \\
(6 \text { meses })\end{array}$ & $\begin{array}{l}\text { Pacientes } \\
\text { (19) }\end{array}$ & $\begin{array}{c}\text { Lumbar } \\
\text { (L1-L2/L2-L3/L5-S1) }\end{array}$ & $\begin{array}{l}\text { Terapia espinal } \\
\text { manipulativa }\end{array}$ & $\begin{array}{l}\text { Disminución del dolor interdiscal en una } \\
\text { sola sesión terapéutica. }\end{array}$ \\
\hline $\begin{array}{l}\text { Fortin, Gibbons, Videman y Battié } \\
\text { (2015). }\end{array}$ & USA & $\begin{array}{l}\text { Pre-experimental } \\
(1,5 \text { y } 15 \text { años })\end{array}$ & $\begin{array}{c}\text { Gemelos } \\
(99)\end{array}$ & $\begin{array}{c}\text { Lumbar } \\
\text { (L2-L3/L5-S1) }\end{array}$ & $\begin{array}{c}\text { Participación regular en } \\
\text { deportes }\end{array}$ & $\begin{array}{l}\text { Las personas físicamente más activas } \\
\text { presentaron umbrales de dolor más bajos. }\end{array}$ \\
\hline Hansen, Nasypany y Baker (2017). & USA & $\begin{array}{l}\text { Estudios de casos } \\
(4 \text { sesiones })\end{array}$ & $\begin{array}{l}\text { Pacientes } \\
(2)\end{array}$ & $\begin{array}{c}\text { Lumbar } \\
\text { (L5 y Sacro) }\end{array}$ & $\begin{array}{c}\text { Técnica de energía } \\
\text { muscular y MyoKinesthetic }\end{array}$ & $\begin{array}{l}\text { Utilización de dos métodos de activación } \\
\text { muscular con disminución del dolor. }\end{array}$ \\
\hline Krych et al. (2012). & USA & $\begin{array}{l}\text { Pre-experimental } \\
\text { (7 años) }\end{array}$ & $\begin{array}{c}\text { Futbolistas (NFL) } \\
(17)\end{array}$ & Lumbar & $\begin{array}{c}\text { Inyecciones epidurales de } \\
\text { esteroides }\end{array}$ & $\begin{array}{l}\text { En diversos casos el efecto de la inyección } \\
\text { no dio resultados favorables. }\end{array}$ \\
\hline Kuai et al. (2017). & China & $\begin{array}{l}\text { Estudio de casos } \\
(1 \mathrm{mes})\end{array}$ & $\begin{array}{c}\text { Pacientes } \\
\text { (33) }\end{array}$ & $\begin{array}{l}\text { Lumbar } \\
\text { (L1/L5) }\end{array}$ & Subir desniveles & $\begin{array}{l}\text { Impacto negativo sobre la carga espinal y } \\
\text { aumento de la carga sobre la musculatura. }\end{array}$ \\
\hline Lee y McGill (2015). & Canadá & $\begin{array}{l}\text { Pre-experimental } \\
(6 \text { Semanas })\end{array}$ & $\begin{array}{l}\text { Atletas } \\
(24)\end{array}$ & $\begin{array}{l}\text { Lumbar } \\
\text { (L4-L5) }\end{array}$ & $\begin{array}{c}\text { Ejercicio físico isométrico y } \\
\text { dinámico }\end{array}$ & $\begin{array}{l}\text { El núcleo discal aumentó de grosor } \\
\text { dándole a la columna más estabilidad. }\end{array}$ \\
\hline Mai et al. (2016). & USA & $\begin{array}{l}\text { Pre-experimental } \\
\text { (2 años) }\end{array}$ & $\begin{array}{l}\text { Futbolistas (NFL) } \\
\text { (559) }\end{array}$ & Lumbar & Terapia ortopédica & $\begin{array}{l}\text { Tras la intervención ortopédica el } 79,4 \% \\
\text { de los atletas continuaron en el fútbol. }\end{array}$ \\
\hline Minhas, Kester y Hsu (2016). & USA & $\begin{array}{l}\text { Estudios de casos } \\
\quad(2-3 \text { años })\end{array}$ & $\begin{array}{l}\text { Jugadores Baloncesto } \\
\text { (61) }\end{array}$ & Lumbar & $\begin{array}{c}\text { Operación quirúrgica } \\
\text { RTP }\end{array}$ & $\begin{array}{l}\text { Aquellos que jugadores que fueron } \\
\text { operados terminaron jugando menos } \\
\text { partidos que los sometidos a masajes. }\end{array}$ \\
\hline Nagashima et al. (2013). & USA & $\begin{array}{l}\text { Pre-experimental } \\
\quad(2 \text { años })\end{array}$ & $\begin{array}{c}\text { Futbolistas } \\
(160)\end{array}$ & Lumbar & Actividad física & $\begin{array}{l}\text { La práctica de fútbol durante dos años } \\
\text { provocó degeneración del núcleo pulposo. }\end{array}$ \\
\hline $\begin{array}{l}\text { Schroeder, McCarthy, Micev, } \\
\text { Terry y Hsu (2013). }\end{array}$ & USA & $\begin{array}{l}\text { Pre-experimental } \\
\quad(2 \text { años })\end{array}$ & $\begin{array}{c}\text { Jugadores de Hockey } \\
\text { (87) }\end{array}$ & Lumbar & $\begin{array}{c}\text { Terapia física } \\
\text { Cirugía } \\
\text { Fusión en un nivel } \\
\end{array}$ & $\begin{array}{l}\text { Aquellos jugadores que fueron tratados } \\
\text { por terapia fisica obtuvieron mejores } \\
\text { niveles de rendimiento que los operados. }\end{array}$ \\
\hline Schroeder et al. (2015). & USA & $\begin{array}{l}\text { Cuasi-experimental } \\
\text { (8 años) }\end{array}$ & $\begin{array}{c}\text { Futbolistas } \\
323\left(\mathrm{GC}^{*}\right)-253\left(\mathrm{GE}^{*}\right) \\
\end{array}$ & $\begin{array}{c}\text { Lumbar } \\
\text { (L4-L5/ L5-S1) }\end{array}$ & Juegos físico-deportivos & $\begin{array}{l}\text { Los deportistas con daños lumbares } \\
\text { tuvieron un rendimiento menor. }\end{array}$ \\
\hline Svensson, Wendt y Thomeé (2014). & Suecia & $\begin{array}{l}\text { Pre-experimental } \\
(1,5 />24 \text { meses })\end{array}$ & $\begin{array}{c}\text { Participantes } \\
(41)\end{array}$ & $\begin{array}{c}\text { Lumbar } \\
\text { (L3-L4/L4-L5/L5-S1) }\end{array}$ & MDT & $\begin{array}{l}\text { El tratamiento físico terapéutico redujo los } \\
\text { niveles de dolor }\end{array}$ \\
\hline $\begin{array}{l}\text { Svensson, Wendt, Thomeé y } \\
\text { Danielson (2013). }\end{array}$ & Suecia & $\begin{array}{l}\text { Pre-experimental } \\
\quad \text { (3 años) }\end{array}$ & $\begin{array}{l}\text { Pacientes } \\
(20)\end{array}$ & Lumbar & $\begin{array}{l}\text { Tratamiento fisioterapéutico } \\
\text { Operación quirúrgica }\end{array}$ & $\begin{array}{l}\text { El tratamiento fisioterapéutico proporción } \\
\text { sensaciones de bienestar } 3 \text { años después. }\end{array}$ \\
\hline $\begin{array}{l}\text { Thackeray, Fritz, Lurie, Zhao y } \\
\text { Weinstein (2017). }\end{array}$ & USA & $\begin{array}{l}\text { Cuasi-experimental } \\
(3 \text { meses-1año })\end{array}$ & $\begin{array}{c}\text { Pacientes } \\
96(\mathrm{GC})-73(\mathrm{GE})\end{array}$ & $\begin{array}{l}\text { Lumbar } \\
\text { (L5-S1) }\end{array}$ & Terapia física & $\begin{array}{l}\text { Tras recibir atención de terapia física, los } \\
\text { índices de dolor no disminuyeron. }\end{array}$ \\
\hline $\begin{array}{l}\text { Vieira, Oliva, Rodríguez, Heredia, } \\
\text { Ricard y Almazán (2014). }\end{array}$ & España & $\begin{array}{l}\text { Cuasi- experimental } \\
\text { (8 años) }\end{array}$ & $\begin{array}{c}\text { Participantes } \\
20(\mathrm{GC})-20(\mathrm{GE})\end{array}$ & $\begin{array}{l}\text { Lumbar } \\
\text { (L5-S1) }\end{array}$ & Terapia física & $\begin{array}{l}\begin{array}{l}\text { Mejora a corto plazo del dolor } \\
\text { intervertebral lumbosacra. }\end{array} \\
\end{array}$ \\
\hline
\end{tabular}

Para citar este artículo utilice la siguiente referencia: González-Valero, G.; Ubajo-Jiménez, J.L.; Castro-Sánchez, M.; García-Martínez, I.; Sánchez-Zafra, Mª.. (2019). Prevención y tratamiento de lesiones lumbares con herramientas físico-médicas. Una revisión sistemática. Sportis Sci J, 5 (2), 232-249.

DOI: https://doi.org/10.17979/sportis.2019.5.2.3409

http://revistas.udc.es/ 


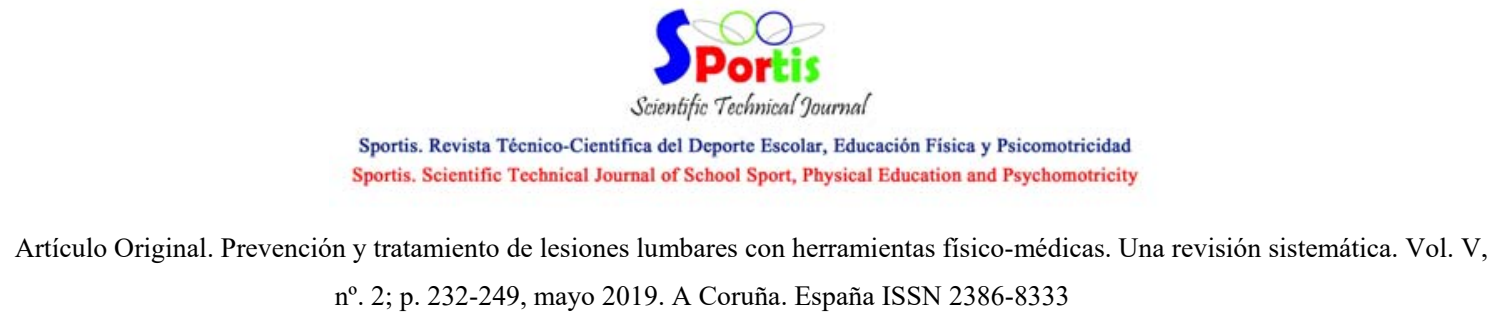

Como se viene indicando, la tabla 2 muestra los resultados extraídos para el análisis del objetivo de estudio bajo la codificación establecida anteriormente, en el que han participado un total de 1907 deportistas y/o sujetos con una actividad física moderada. Se debe resaltar que el $60 \%(\mathrm{~N}=9)$ de los estudios seleccionados para la revisión sistemática ofrecían un diseño metodológico pre-experimental con intervención, así mismo el $20 \%(\mathrm{~N}=3)$ de estas investigaciones, mostraban que su metodología se basaba en el estudio de casos o en tratamientos cuasi-experimentales con grupo control y experimental.

Es de vital importancia destacar los países o territorios donde se han realizado los estudios sobre el tratamiento y prevención de lesiones lumbosacras (L5-S1) en población físicamente activa. Podemos observar que para el análisis de estudios de esta revisión sistemática se han desarrollado en cinco países o territorios (Estados Unidos, China, Canadá, Suecia y España), donde se ha de resaltar los estudios realizados es Estados Unidos, ya que representan el 66,66\% $(\mathrm{N}=11)$ de estudios realizados, aun así, se hace necesario destacar que en España encontramos un artículo de investigación científica relacionado con la temática de estudio.

\section{Discusión}

Los daños en la zona lumbar y la zona del sacro, es uno de los efectos más comunes que se detectan en los deportistas, ocasionados por posiciones incorrectas que terminan por dañar la zona baja de la espalda, debida a la tensión que se genera en los discos vertebrales. Tras realizar una revisión sobre la temática analizada se observa que desde antiguamente se viene utilizando como medio para subsanar esta problemática la cirugía, actualmente se han encontrado múltiples beneficios, sobre esta zona, extraídos de la práctica de ejercicios terapéuticos los cuales contribuyen a disminuir el dolor en los sujetos dañados, así como medio preventivo para evitar su aparición.

Algunos de esos estudios como el desarrollado por Beattie et al., (2014), muestran como mediante la intervención terapéutica sobre 19 participantes con problemas de hernias en los niveles L1-L2, L2-L3, L5-S1, consiguen reducir la tensión de los discos vertebrales, favoreciendo la disminución del dolor, mediante la utilización de ejercicios básicos estáticos y forzados. De la misma manera, Hansen et al., (2017), mediante la aplicación de un método de

Para citar este artículo utilice la siguiente referencia: González-Valero, G.; Ubajo-Jiménez, J.L.; Castro-Sánchez, M.; García-Martínez, I.; SánchezZafra, $M^{\mathrm{a}}$.. (2019). Prevención y tratamiento de lesiones lumbares con herramientas físico-médicas. Una revisión sistemática. Sportis Sci J, 5 (2), $232-249$.

DOI: https://doi.org/10.17979/sportis.2019.5.2.3409

http://revistas.udc.es/ 


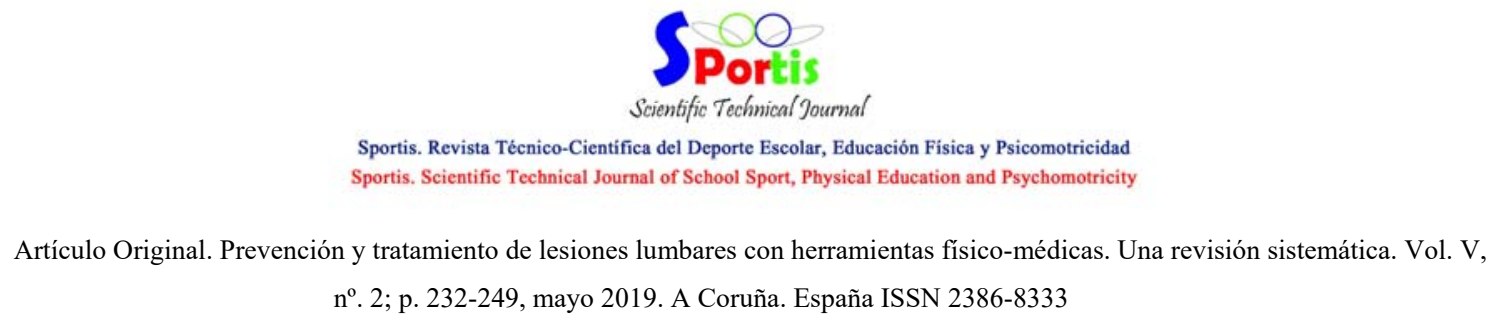

actividad activa y pasiva de músculos y electro-estimulación muscular, obtuvieron resultados muy favorecedores en cuanto a la información de la resolución completa del dolor, y en cuanto al tiempo de rehabilitación (10 sesiones de tratamiento), los cuales anteriormente habían sido sometidos a cirugía obteniendo un resultado fallido (Brody, Baker, Nasypany y May, 2014; Svensson et al., 2014).

Por otro lado, los autores Garvey et al., (2012) y Lee y McGill (2015), pusieron de relieve que los entrenadores que proponían a los deportistas ejercicios a desarrollar, antes de dar comienzo a la sesión, de tipo soporte, como sentadillas o cargar con peso, fortalecimiento de la zona abdominal, así como hacer hincapié en presentar una correcta higiene corporal, ayuda a adquirir una estabilidad vertebral mediante el aumento del grosor del disco intervertebral, lo cual contribuye a una menor acumulación de tensión en la zona lumbar de los sujetos tanto afectados por este tipo de síntomas, como de forma preventiva para aquellos que están sanos.

Sin embargo, otras investigaciones como la de Kuai et al., (2017), en la cual se llevó a cabo una intervención de ejercicios físicos sobre pacientes con daños en la zona lumbar y sujetos sin problemas, tras poner a los participantes a andar en situaciones de desniveles se obtuvieron conclusiones negativas en ambos sujetos, debido a que la naturaleza repetitiva del ejercicio propuesto, más la carga continua, incrementó el riesgo de empeoramiento de hernia discal a nivel lumbar. Tras finalizar el estudio, se les sugirió a algunos pacientes que recibieran un tratamiento quirúrgico para evitar el avance de esta patología en un futuro. Ocurriendo de forma similar en los pacientes del estudio de Thackeray et al. (2017).

Se ha de destacar dentro de los deportes, que los jugadores de fútbol son de los que más trastornos a nivel musculo-esquelético presentan, provocados por causas de colisión, la superficie de juego, las condiciones climáticas, la continua preparación física, los movimientos repetitivos, lo que en ocasiones los lleva a operaciones, las cuales pueden suponer el fin de su carrera (Fortin et al., 2015; González-Valero, Zurita-Ortega, San RománMata, Pérez-Cortés, Puertas-Molero y Chacón-Cuberos, 2018; Nagashima et al. 2013; Schroeder et al. 2015). Mai et al., (2016) realizó un estudio sobre futbolistas afectados tanto a nivel lumbar, como de otras articulaciones como los tobillos, en el cual aplicó un tratamiento

Para citar este artículo utilice la siguiente referencia: González-Valero, G.; Ubajo-Jiménez, J.L.; Castro-Sánchez, M.; García-Martínez, I.; SánchezZafra, $M^{a}$.. (2019). Prevención y tratamiento de lesiones lumbares con herramientas físico-médicas. Una revisión sistemática. Sportis Sci J, 5 (2), $232-249$. DOI: https://doi.org/10.17979/sportis.2019.5.2.3409

http://revistas.udc.es/ 
Artículo Original. Prevención y tratamiento de lesiones lumbares con herramientas físico-médicas. Una revisión sistemática. Vol. V, n. 2; p. 232-249, mayo 2019. A Coruña. España ISSN 2386-8333

ortopédico, mediante el cual obtuvo excelentes resultados, ya que tras desarrollar su intervención la mayoría de los deportistas pudieron volver a retomar su carrera deportiva sin la presencia de dolor.

Otro estudio similar, fue el desarrollado por Minhas et al., (2016), el cual comparó los resultados de jugadores de baloncesto con problemas de hernia discal en la zona lumbar y sacro que habían sido operados y en aquellos que habían recibido un tratamiento físico, mostrando resultados más favorecedores para aquellos que no habían sido tratados quirúrgicamente, ya que de los sujetos con tratamiento terapéutico el 74,9\% pudo retomar su vida normal sin problemas, así como que consiguieron aumentar el número de partidos jugados.

La modalidad deportiva de Hockey obtuvo a su vez resultados similares a los anteriores, en los cuales se desarrolló un estudio comparativo entre jugadores con daños lumbares sometidos a operación y jugadores sometidos a tratamiento terapéutico, los cuales desarrollaron ejercicios para fortalecer la zona abdominal y el tono muscular en general, en cuanto a la vuelta al deporte mostraron los mismos datos de reposición un $85 \%$ en cada caso, pero en cuanto a la comparación de rendimiento y partidos jugados, obtuvieron resultados más favorecedores aquellos sujetos que no habían pasado por tratamiento quirúrgico (Schroeder et al., 2013).

Metodologías como la inyección epidural con esteroides fueron las utilizadas por los investigadores Krych et al., (2012), de las cuales de cada 4 atletas que la recibieron solo dio resultados positivos a uno, los otros tres la adquirieron en diversas ocasiones sin conseguir aliviar la tensión del disco lumbar, el cual les producía un pinzamiento del nervio, llegando al final a necesitar tratamiento quirúrgico y posteriormente a su vez rehabilitación de ejercicios físicos para su correcta recuperación.

En cuanto a las sensaciones de bienestar o malestar en sujetos con hernias lumbares, Svensson et al. (2013), llevó a cabo una encuesta abierta con el fin de comparar las emociones de sujetos que habían sido tratados de forma quirúrgica y con los que habían recibido un tratamiento fisioterapéutico, tras pasar un periodo de tiempo de tres años. Poniendo de relieve que aquellos sujetos que se habían sometido a operación presentaban sentimientos de

Para citar este artículo utilice la siguiente referencia: González-Valero, G.; Ubajo-Jiménez, J.L.; Castro-Sánchez, M.; García-Martínez, I.; SánchezZafra, $M^{\mathrm{a}}$.. (2019). Prevención y tratamiento de lesiones lumbares con herramientas físico-médicas. Una revisión sistemática. Sportis Sci J, 5 (2), $232-249$. 


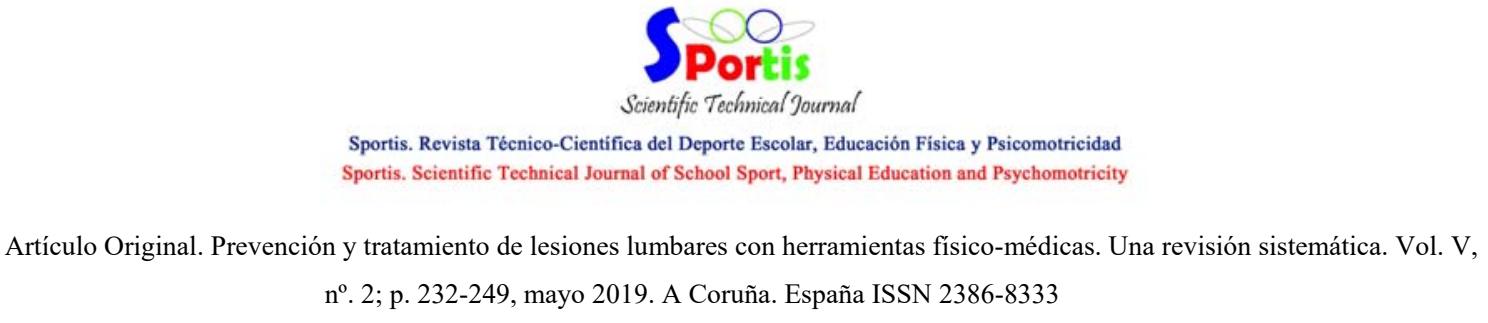

malestar, mientras que el resto presentaban mayoritariamente efectos de bienestar (Vieira et al., 2014).

\section{Conclusiones}

El propósito de esta revisión sistemática, ha sido analizar los beneficios que reporta la actividad física en la mejora y prevención de hernias en la columna vertebral a nivel lumbar y sacro, tomando como punto de referencia la producción científica que abarca esta temática en los últimos cinco años, así como aquellas que han utilizado intervenciones mostrando las repercusiones tanto positivas como negativas que estos han desencadenado en los sujetos.

En relación a la producción de artículos, se hace necesario destacar que, en los últimos años, se ha pasado de utilizar únicamente, para el tratamiento de daños en los discos vertebrales, intervenciones quirúrgicas, a probar diversas estrategias relacionadas con la práctica de ejercicios controlados por especialistas, poniendo de manifiesto que estas últimas estrategias reportan mayores beneficios, tanto a nivel físico como emocional en los pacientes, que el pasar por un proceso quirúrgico.

A su vez, la temática que se aborda, ha sido desarrollada en diversos países teniendo una gran repercusión USA, ya que es el territorio en el cual se ha identificado una mayor producción sobre el tema a tratar, sin embargo para España solo se ha detectado una intervención, por lo que se hace necesario tomar consciencia sobre la gran población que se ve afectada por esta patología e intervenir mediante métodos físico deportivos, los cuales han mostrado tener un gran impacto sobre la reducción del dolor sobre la zona lumbar y saco.

A su vez, se pone en relieve que la práctica de ejercicio relacionada con la tonificación muscular, el fortalecer la zona abdominal y adquirir una correcta postura higiénica, entre otras, favorece la estabilización de la parte de la columna vertebral y le proporciona una mayor elasticidad, siendo esta de gran importancia para los deportistas, ya que se constituye como un medio para prevenir lesiones a nivel lumbar y de sacro, mayoritariamente en aquellas modalidades deportivas de contacto, de carga de peso y de repetición continuo de un mismo gesto.

En definitiva, la realización de ejercicio físico influye positivamente en la prevención y tratamiento de hernias de la columna vertebral, siendo este método más representativo en

Para citar este artículo utilice la siguiente referencia: González-Valero, G.; Ubajo-Jiménez, J.L.; Castro-Sánchez, M.; García-Martínez, I.; SánchezZafra, $M^{a}$.. (2019). Prevención y tratamiento de lesiones lumbares con herramientas físico-médicas. Una revisión sistemática. Sportis Sci J, 5 (2), $232-249$. DOI: https://doi.org/10.17979/sportis.2019.5.2.3409

http://revistas.udc.es/ 


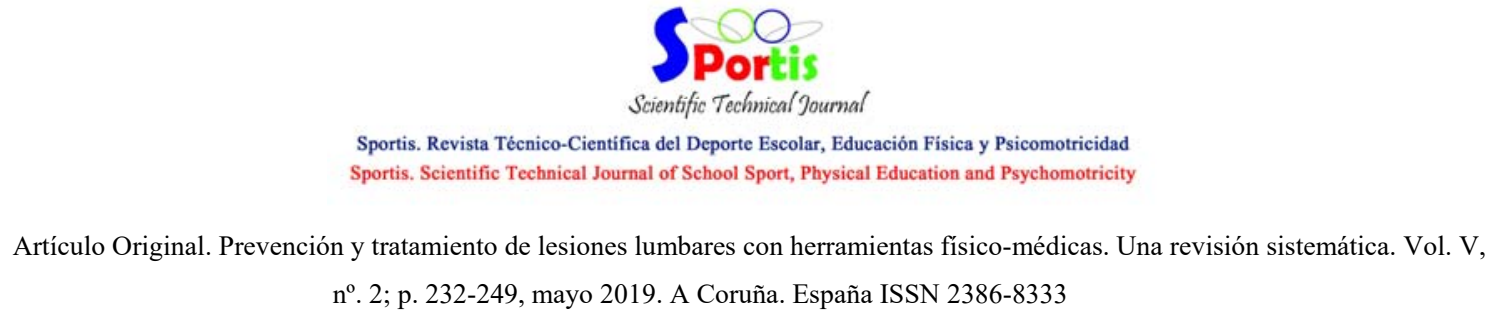

cuanto a la cura y vuelta a la vida normal que el tratamiento quirúrgico, además contribuye a que los sujetos adquieran un mayor control de su cuerpo, proporcionándoles tanto seguridad a nivel esquelético- muscular, como a nivel de reducción de la tensión intervertebral, disminuyendo a su vez la sensación de dolor.

Por lo tanto, como perspectivas futuras se invita a realizar intervenciones, tanto en sujetos dañados, como sanos, para seguir avanzando en métodos que actúen como pautas de prevención y de tratamiento ante estos problemas y consigan reducir los niveles de dolor (L5S1) en los sujetos, que les garantice su continuidad en el ámbito deportivo.

\section{Referencias bibliográficas}

Al-Najjim, M., Shah, R., Rahuma, M., y Gabbar, O. A. (2018). Lumbar facet joint injection in treating low back pain: Radiofrequency denervation versus SHAM procedure. $\begin{array}{llll}\text { Systematic } & \text { review. Journal of } & \text { orthopaedics, 15(1), }\end{array}$ https://doi.org/10.1016/j.jor.2017.10.001

Atabey, C., Eroğlu, A., Topuz, A., Velioğlu, M., y Demircan, M. (2014). Lumbar disc herniation in a patient with congenital vertebral body anomaly: a case report. Korean Journal of Spine, 11(4), 245-248. https://doi.org/10.14245/kjs.2014.11.4.245

Beattie, P. Butts, R., Donley, J. y Liuzzo, D. (2014). The within-session change in low back pain intensity following spinal manipulative therapy is related to differences in diffusion of water in the intervertebral discs of the upper lumbar spine and L5-S1. Journal of orthopaedic \& sports physical therapy, 44(1), 19-29. https://doi.org/10.2519/jospt.2014.4967

Beaudette, S. M., Larson, K. J., Larson, D. J., y Brown, S. H. (2016). Low back skin sensitivity has minimal impact on active lumbar spine proprioception and stability in healthy adults. Experimental brain research, 234(8), 2215-2226. https://doi.org/10.1007/s00221-016$\underline{4625-5}$

Bozkurt, S., Aktekin, L., Ugurlu, F. G., Balci, S., Sezer, N. y Akkus, S. (2017). An Unusual Cause of Myelopathy: Ochronotic Spondyloarthropathy With Positive Hla B27. American Journal of Physical Medicine and Rehabilitation,96(11), 206-209. https://doi.org/10.1097/phm.0000000000000727

Para citar este artículo utilice la siguiente referencia: González-Valero, G.; Ubajo-Jiménez, J.L.; Castro-Sánchez, M.; García-Martínez, I.; SánchezZafra, $M^{a}$.. (2019). Prevención y tratamiento de lesiones lumbares con herramientas físico-médicas. Una revisión sistemática. Sportis Sci J, 5 (2), 232-249. DOI: https://doi.org/10.17979/sportis.2019.5.2.3409

http://revistas.udc.es/ 
Artículo Original. Prevención y tratamiento de lesiones lumbares con herramientas físico-médicas. Una revisión sistemática. Vol. V, n. 2; p. 232-249, mayo 2019. A Coruña. España ISSN 2386-8333

Brody, K., Baker, R., Nasypany, A. y May, J. (2014). Treatment of chronic low back pain using the Myokinesthetic System: Part 2. Int J Athl Ther Train, 20(5), 22-28. https://doi.org/10.1097/10.1123/ijatt.2014-0132

Fortin, M., Gibbons, L. E., Videman, T. y Battié, M. C. (2015). Do variations in paraspinal muscle morphology and composition predict low back pain in men? Scandinavian journal of medicine and science in sports, 25(6), 880-887. https://doi.org/10.1111/sms.12301

Garvey, J., Read, J., y Turner, A. (2010). Sportsman hernia: what can we do?. Hernia, 14(1), 17-25. https://doi.org/10.1007/s10029-009-0611-1

González-Valero, G., Zurita-Ortega, F., Puertas-Molero, P., Espejo-Garcés, T., ChacónCuberos, R., y Castro-Sánchez, M. (2017). Influencia de los factores sedentarios (dieta y videojuegos) sobre la obesidad en escolares de Educación Primaria. ReiDoCrea: Revista electrónica de investigación y docencia creativa, 6, 120-129. https://doi.org/10.6018/300491

González-Valero, G., Zurita-Ortega, F., San Román-Mata, S., Pérez-Cortés, A. J., Puertas-Molero, P., y Chacón-Cuberos, R. (2018). Analysis of aerobic capacity as an essential quality of students' physical condition: A systematic review. Retos, 34, 395-402.

Hansen, J. A., Nasypany, A. M. y Baker, R. T. (2017). Treatment Utilizing a Muscle Energy Technique and the MyoKinesthetic System on Patients With a Diagnosed Disc Injury. International Journal of Athletic Therapy \& Training, 22(4), 6-12. https://doi.org/10.1123/ijatt.2016-0013

Hernández, G. A., y Zamora-Salas, J. D. (2017). Exercise as a treatment for low back pain management. Revista de Salud Pública, 19(1), 123-128.

Herndon, C. L., Horodyski, M., y Vincent, H. K. (2017). Acute effects of anesthetic lumbar spine injections on temporal spatial parameters of gait in individuals with chronic low back pain: A pilot study. Gait \& posture, 58, 369-373. https://doi.org/10.1016/j.gaitpost.2017.08.016

Hutton, B., Salanti, G., Caldwell, D. M., Chaimani, A., Schmid, C. H., Cameron, C., ... y Mulrow, C. (2015). The PRISMA Extension Statement for Reporting of Systematic Reviews Incorporating Network Meta-analyses of Health Care Interventions: Checklist and

Para citar este artículo utilice la siguiente referencia: González-Valero, G.; Ubajo-Jiménez, J.L.; Castro-Sánchez, M.; García-Martínez, I.; SánchezZafra, $M^{\mathrm{a}}$.. (2019). Prevención y tratamiento de lesiones lumbares con herramientas físico-médicas. Una revisión sistemática. Sportis Sci J, 5 (2), $232-249$. DOI: https://doi.org/10.17979/sportis.2019.5.2.3409

http://revistas.udc.es/ 
Artículo Original. Prevención y tratamiento de lesiones lumbares con herramientas físico-médicas. Una revisión sistemática. Vol. V, $n^{\circ}$. 2; p. 232-249, mayo 2019. A Coruña. España ISSN 2386-8333

Explanations PRISMA Extension for Network Meta-analysis. Annals of internal medicine, 162(11), 777-784. https://doi.org/10.7326/m14-2385

Kovacs, F., y Arana, E. (2016). Patología degenerativa en la columna lumbar. Radiología, 58, 26-34. https://doi.org/10.1016/j.rx.2015.12.004

Krych, A. J., Richman, D., Drakos, M., Weiss, L., Barnes, R., Cammisa, F. y Warren, R. F. (2012). Epidural steroid injection for lumbar disc herniation in NFL athletes. Medicine and Science in Sports and Exercise, 44(2), 193-198. https://doi.org/10.1249/mss.0b013e31822d7599

Kuai, S., Liao, Z., Zhou, W., Guan, X., Ji, R., Zhang, R., Guo, D. y Liu, W. (2017). The Effect of Lumbar Disc Herniation on Musculoskeletal Loadings in the Spinal Region During Level Walking and Stair Climbing. Medical Science Monitor: International Medical Journal of Experimental and Clinical Research, 23, 3869-3877. https://doi.org/10.12659/msm.903349

Lee, B., y McGill, S. (2015). Effect of long-term isometric training on core/torso stiffness. The Journal of Strength and Conditioning Research,29(6), 1515-1526. https://doi.org/10.1519/jsc.0000000000000740

Lopes, S., Correia, C., Félix, G., Lopes, M., Cruz, A., y Ribeiro, F. (2017). Immediate effects of Pilates based therapeutic exercise on postural control of young individuals with non-specific low back pain: A randomized controlled trial. Complementary therapies in medicine, 34, 104-110. https://doi.org/10.1016/j.ctim.2017.08.006

Mai, H., Alvarez, A., Freshman, R., Chun, D., Minhas, S., Patel, A., ... y Hsu, W. (2016). The NFL Orthopaedic Surgery Outcomes Database (NO-SOD) The Effect of Common Orthopaedic Procedures on Football Careers. The American journal of sports medicine, 44(9), 2255-2262. https://doi.org/10.1177/0363546516651426

Minhas, S., Kester, B. y Hsu, W. K. (2016). Outcomes after lumbar disc herniation in the National Basketball Association. Sports health, 8(1), 43-49. https://doi.org/10.1177/1941738115608361

Nagashima, M., Abe, H., Amaya, K., Matsumoto, H., Yanaihara, H., Nishiwaki, Y., Toyama, A. y Matsumoto, M. (2013). Risk factors for lumbar disc degeneration in high

Para citar este artículo utilice la siguiente referencia: González-Valero, G.; Ubajo-Jiménez, J.L.; Castro-Sánchez, M.; García-Martínez, I.; SánchezZafra, $M^{a}$.. (2019). Prevención y tratamiento de lesiones lumbares con herramientas físico-médicas. Una revisión sistemática. Sportis Sci J, 5 (2), 232-249. DOI: https://doi.org/10.17979/sportis.2019.5.2.3409 
Artículo Original. Prevención y tratamiento de lesiones lumbares con herramientas físico-médicas. Una revisión sistemática. Vol. V, nº. 2; p. 232-249, mayo 2019. A Coruña. España ISSN 2386-8333

school American football players: a prospective 2-year follow-up study. The American journal of sports medicine, 41(9), 2059-2064. https://doi.org/10.1177/0363546513495173

Pantoja, S. (2012). Lesiones de la columna lumbar en el deportista. Revista médica clínica las condesas, 23(3), 275-282. https://doi.org/10.1016/s0716-8640(12)70311-5

Pardo, G. B., Girbés, E. L., Roussel, N. A., Izquierdo, T. G., Penick, V. J., y Martín, D. P. (2018). Pain Neurophysiology Education and Therapeutic Exercise for Patients With Chronic Low Back Pain: A Single-Blind Randomized Controlled Trial. Archives of physical medicine and rehabilitation, 99(2), 338-347. https://doi.org/10.1016/j.apmr.2017.10.016

Schroeder, G. Lynch, T. Gibbs, D. Chow, I., LaBelle, M., Patel, A. Jason, S., Wellington, H. y Nuber, G. W. (2015). Pre-existing lumbar spine diagnosis as a predictor of outcomes in National Football League athletes. The American journal of sports medicine, 43(4), 972-978. https://doi.org/10.1177/0363546514562548

Schroeder, G. McCarthy, K. Micev, A. Terry, M. y Hsu, W. (2013). Performancebased outcomes after nonoperative treatment, discectomy, and/or fusion for a lumbar disc herniation in National Hockey League athletes. The American journal of sports medicine, 41(11), 2604-2608. https://doi.org/10.1177/0363546513499229

Su, C. A., Kusin, D. J., Li, S. Q., Ahn, U. M., y Ahn, N. U. (2018). The Association Between Body Mass Index and the Prevalence, Severity, and Frequency of Low Back Pain: Data From the Osteoarthritis Initiative. Spine, 43(12), 848-852. https://doi.org/10.1097/brs.0000000000002601

Svensson, G. Wendt, G. y Thomeé, R. (2014). A structured physiotherapy treatment model can provide rapid relief to patients who qualify for lumbar disc surgery: a prospective cohort study. Journal of rehabilitation medicine, 46(3), 233-240. https://doi.org/10.2340/16501977-1255

Svensson, G. L., Wendt, G. K., Thomeé, R. y Danielson, E. (2013). Patients' experience of health three years after structured physiotherapy or surgery for lumbar disc herniation. Journal of rehabilitation medicine, 45(3), 293-299. https://doi.org/10.2340/16501977-1105

Para citar este artículo utilice la siguiente referencia: González-Valero, G.; Ubajo-Jiménez, J.L.; Castro-Sánchez, M.; García-Martínez, I.; SánchezZafra, $M^{a}$.. (2019). Prevención y tratamiento de lesiones lumbares con herramientas físico-médicas. Una revisión sistemática. Sportis Sci J, 5 (2), $232-249$. DOI: https://doi.org/10.17979/sportis.2019.5.2.3409 


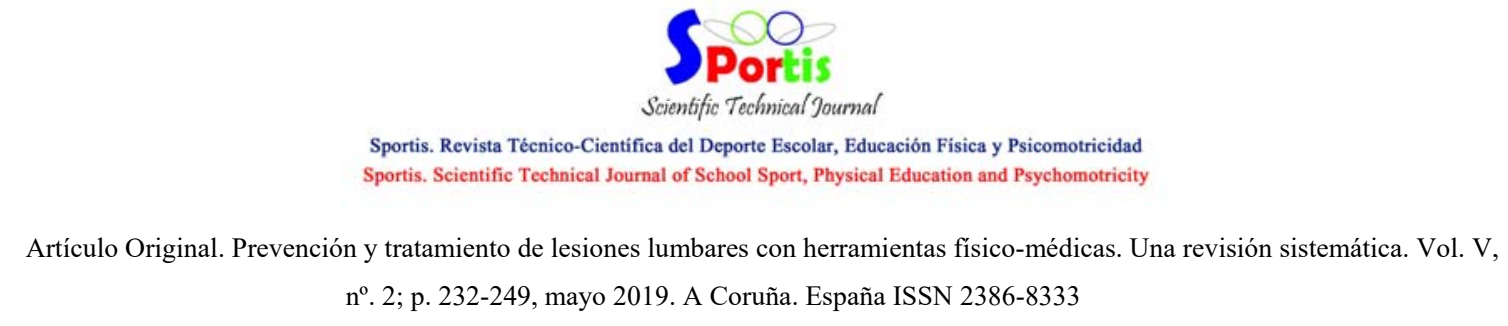

Thackeray, A., Fritz, J. M., Lurie, J. D., Zhao, W. y Weinstein, J. N. (2017). Nonsurgical treatment choices by individuals with lumbar intervertebral disc herniation in the United States: associations with long-term outcomes. American journal of physical medicine and rehabilitation, 96(8), 557-564. https://doi.org/10.1097/phm.0000000000000685

Vieira, F., Oliva, Á., Rodríguez, C., Heredia, A., Ricard, F. y Almazán, G. (2014). Short-term effect of spinal manipulation on pain perception, spinal mobility, and full height recovery in male subjects with degenerative disk disease: a randomized controlled trial. Archives of physical medicine and rehabilitation,95(9), 1613-1619. https://doi.org/10.1016/j.apmr.2014.05.002

Winslow, J., Getzin, A., Greenberger, H., y Silbert, W. (2017). Fatty Infiltrate of the Lumbar Multifidus Muscles Predicts Return to Play in Young Athletes With Extension-Based Low Back Pain. Clinical journal of sport medicine: official journal of the Canadian Academy of Sport Medicine, 1, 1-6. https://doi.org/10.1097/jsm.0000000000000483

Zurita-Ortega, F., Olmo-Extremera, M., Cachón-Zagalaz, J., Castro-Sánchez, M., Ruano-Hermoso, B., y Zurita, N. (2015). Relaciones entre lesiones deportivas y parámetros de nivel, fase y modalidad deportiva relations between sports injuries and level parameters, phase and type of sport. Journal of Sport and Health Research, 7(3), 215-228. https://doi.org/10.12800/ccd.v12i35.885

Para citar este artículo utilice la siguiente referencia: González-Valero, G.; Ubajo-Jiménez, J.L.; Castro-Sánchez, M.; García-Martínez, I.; SánchezZafra, $M^{a}$.. (2019). Prevención y tratamiento de lesiones lumbares con herramientas físico-médicas. Una revisión sistemática. Sportis Sci J, 5 (2), $232-249$. DOI: https://doi.org/10.17979/sportis.2019.5.2.3409 http://revistas.udc.es/ 